\title{
RAZONANDO CON COLORES \\ (Una aproximación a la lógica intuicionista)
}

\author{
Jorge Páez $O^{*}$ \\ Carlos Luque A. * \\ Alberto Donado N. *
}

\begin{abstract}
We present an intuitive approach to Intuitionist logic based on the notion of $\underline{n}$-paintings which generalizes the notion of sets; using several tonalities of a color, we construct operations between $\underline{n}$-paintings that can be interpreted in terms of propositions and logical reasoning based on Heyting algebras.
\end{abstract}

\section{INTRODUCCIÓN}

La lógica clásica, en cuya construcción intervienen proposiciones que sólo pueden tomar dos valores de verdad (Verdadero y Falso), ha sido utilizada para la elaboración de la teoría de conjuntos, piedra fundamental para el desarrollo de la matemática moderna y como paradigma para la fundamentación teórica de las ciencias.

Para representar conjuntos suelen utilizarse los diagramas de Euler-Venn, en los que a cada punto de un rectángulo que representa el universo, se le asigna un color para distinguir los puntos que representan los elementos que pertenecen a un conjunto y un color para los elementos que no pertenecen a él, el cual normalmente es el fondo del papel.

Existen lógicas con más de dos valores de verdad [3] y diferentes formas para su construcción [1], [2] que están siendo consideradas como herramientas para los desarrollos tecnológicos actuales [6], lo que hace indispensable facilitar el acercamiento a las mismas y su pronta inclusión en los currículos de las matemáticas escolares con el fin de familiarizar a los estudiantes con otras formas de razonamiento lógico.

La lógica intuicionista, por formar parte de los últimos desarrollos de la matemática es presentada de forma axiomática y exige de bastantes prerrequisitos para su comprensión. Nuestra intención es lograr una aproximación intuitiva, partiendo de la noción de pintura (generalización de la noción de conjunto) como interpretación de la realidad para llegar a las leyes de una lógica asociada a un álgebra de Heyting lineal, [4] proceso inverso al que se utiliza con frecuencia en los textos de matemáticas [9] en donde a partir de la lógica clásica y sus leyes se llega a establecer las propiedades de pinturas de dos colores, que corresponden a los conjuntos.

\footnotetext{
*Profesores Universidad Pedagógica Nacional. 
Dentro del concepto de pintura que presentamos en el presente articulo, deberían incluirse varios colores y una forma de ordenarlos, sin embargo esto no lo consideramos necesario, debido a que con varias tonalidades de un mismo color basta para ejemplificar todas las construcciones que se harían en un caso más general y pueden dar una buena aproximación a las interpretaciones de la realidad.

\section{2. $\underline{n}$-pinturas}

Comencemos con una gama de $n$ tonalidades del mismo color ordenados ascendentemente del más oscuro al más claro que representaremos por $\mathrm{H}$ y con una hoja pintada de la tonalidad más clara disponible, que notaremos $\mathrm{X}$, la cual nos servirá como universo de referencia.

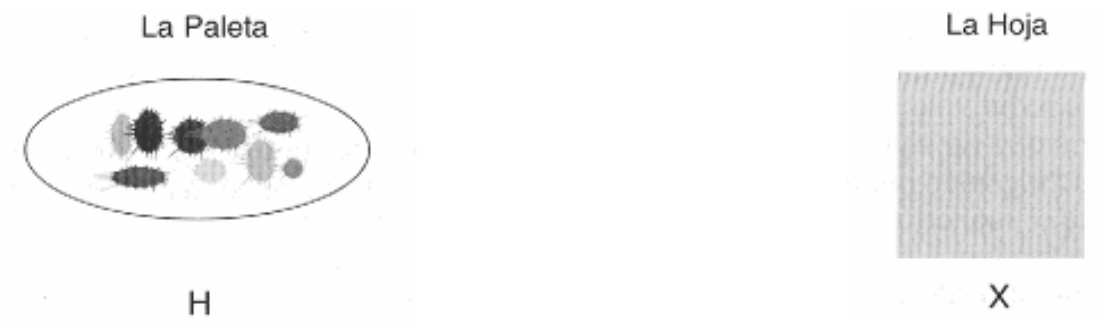

Figura 1.

El orden en $\mathrm{H}$ podemos ponerlo en correspondencia con el orden del conjunto

$$
\{0,1,2,3, \ldots(n-1)\}
$$

como lo muestra el siguiente esquema para el caso de 9 tonalidades de grises:

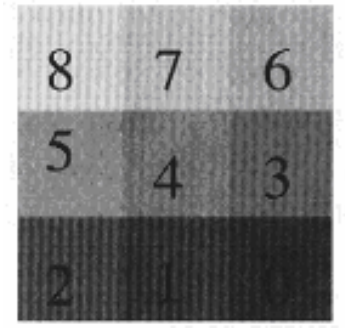

Figura 2.

La parte izquierda de la figura 3 se ha construido con estas tonalidades, haciendo una cuadrícula en $\mathrm{X}$ y asociando por sencillez a cada cuadro uno de los colores de $\mathrm{H}$. Por supuesto el dibujo será más cercano a la realidad, cuanto más fina sea la cuadrícula y cuanto mayor sea el número de tonalidades seleccionadas.

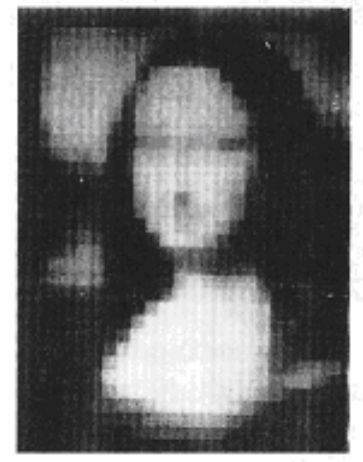

9-pintura

Figura 3.

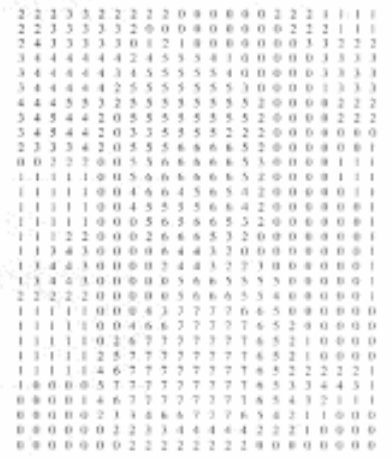

9-predicado 
Al resultado de este proceso de asignación de colores de $\mathrm{H}$ a cada punto del universo $X$ lo llamaremos una $\underline{n}$-pintura.

Notemos que no es necesario que todas las n-pinturas tengan todas las tonalidades, de hecho puede haber $\underline{n}$-pinturas con una sola de las tonalidades disponibles.

\section{Construcción de $\underline{n}$-pinturas: $\underline{n}$-predicados}

Inicialmente generaremos $\underline{n}$-pinturas mediante dos procedimientos básicos:

Una primera forma consiste en partir de un modelo, que bien puede ser un objeto real o mental, plasmado en la hoja a la manera de los pintores, con las limitaciones propias de la gama de colores seleccionados.

Otra forma de lograrlo es disponer de una instrucción o un conjunto de instrucciones que nos indique como llenar la hoja, esto es, como asignar a cada punto de nuestro universo (X) una única tonalidad de las inicialmente seleccionadas $(H)$. A estas instrucciones las llamaremos $\underline{n}$-predicados. Un ejemplo de ello se presenta en la parte derecha de la figura 3.

Queda entonces definida una función entre el conjunto de $n$-predicados, que podríamos identificar con el conjunto de funciones de $\mathrm{X}$ en $\mathrm{H}$ (notado $\mathrm{H}^{\bar{X}}$ ), y el conjunto de las $\underline{n}$-pinturas.

Algunos ejemplos de $\underline{3}$-pinturas son:

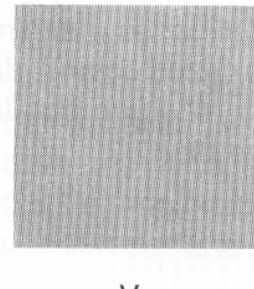

V

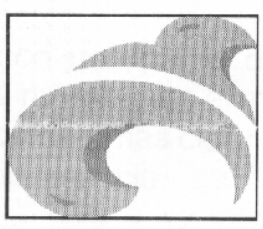

J

$\mathrm{F}$

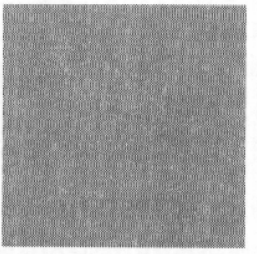

A

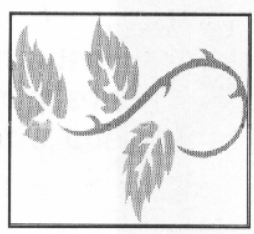

K

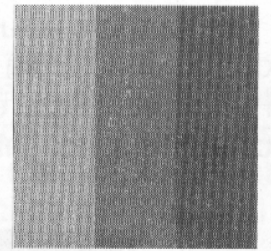

B

Figura 4.

Resaltamos las $\underline{3}$-pinturas marcadas con $\mathrm{V}$ y $\mathrm{F}$, asociadas a los $\underline{3}$-predicados "asignar a cada punto el color más claro y "asignar a cada punto el color más oscuro", que dejan la hoja de un solo color, pues serán de particular importancia para los desarrollos que siguen.

Estas $\underline{3}$-pinturas tienen una generalización, cualquiera que sea la selección de $n$ tonalidades, pues siempre va a haber una $\underline{n}$-pintura con la tonalidad más clara en todos 
los puntos, que llamaremos $\mathrm{V}$ y una con la tonalidad más oscura en todos sus puntos, que llamaremos $\mathrm{F}$.

Ejemplos de 2-pinturas, que bien pudieran verse como diagramas de Euler Venn son:

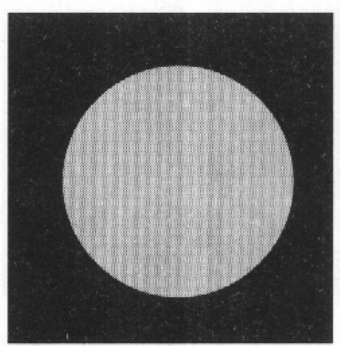

S

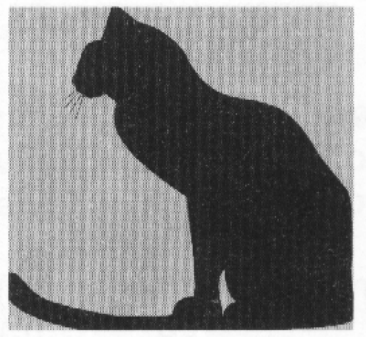

$\mathrm{M}$

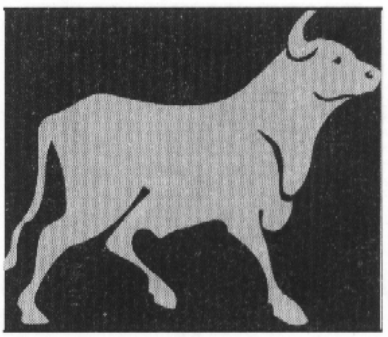

$\mathrm{N}$

Figura 4

La primera 2-pintura, más cercana a nuestra tradición escolar, representa los elementos que forman un conjunto A (zona clara) y los que forman su complemento (zona oscura). Es así como en la figura $5 \mathrm{~N}$ la zona clara muestra los puntos que pertenecen a una representación de una vaca y en la $5 \mathrm{M}$ los que no pertenecen a la representación de un gato (aunque tiene toda la apariencia de gato).

\section{Orden entre $\underline{n}$-pinturas}

El conjunto de las tonalidades $\mathrm{H}$ se puede considerar como un conjunto ordenado por la relación ser mas oscuro o igual, orden que permite establecer una relación de contenencia entre $\underline{n}$-pinturas, de la misma manera como en la teoría de conjuntos, el orden existente en el conjunto $\underline{2}=\{0,1\}$ permite definir la relación de contenencia entre conjuntos [7].

El mecanismo consiste en utilizar el conectivo implica $(\rightarrow$ ) de la lógica bivalente, de la siguiente manera: se dice que la frase "un conjunto A está contenido en otro conjunto B" es verdadera, si la frase "para todo $x \in X,(x \in A \rightarrow x \in B)$ " es verdadera, lo cual se obtiene cuando el valor de verdad de la frase $(x \in A$ ) (asumiendo 0 como falso y 1 como verdadero) es menor o igual que el valor de verdad de la frase $(x \in B)$.

La frase "para todo $x \in X,(x \in A \rightarrow x \in B)$ tiene el valor de verdad de $(x \in B)$ en otro caso. Esto es, ella es falsa, cuando el valor de verdad de $(x \in A)$ sea mayor.

Esta idea puede traducirse en términos de colores en las 2-pinturas, diciendo que el color de cada punto en la 2-pintura asociada con A, sea más oscuro o igual que el color en la $\underline{n}$-pintura asociada con $\mathrm{B}$. Lo cual generalizado a $\underline{n}$-pinturas adquiere la siguiente forma:

La $\underline{n}$-pintura $\mathrm{A}$ está contenida en la $\underline{n}$-pintura $\mathrm{B}$, notado $\mathrm{A} \leq \mathrm{B}$ si cada punto en la $\underline{n}$-pintura A es más oscuro o igual que en la $\underline{n}$-pintura $B$.

La siguiente figura ilustra contenencias entre 4-pinturas: 


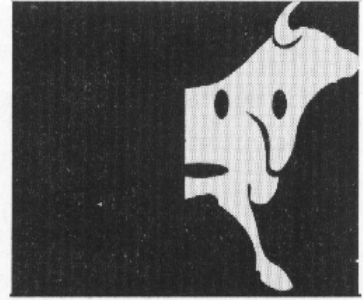

M

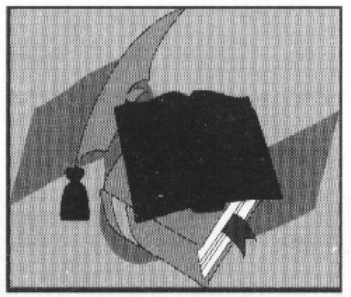

$\mathrm{N}$

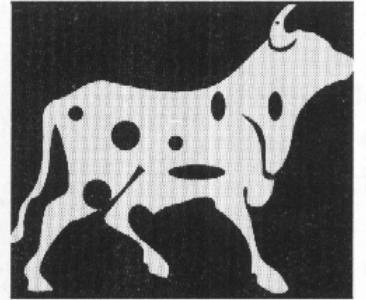

$\mathrm{P}$

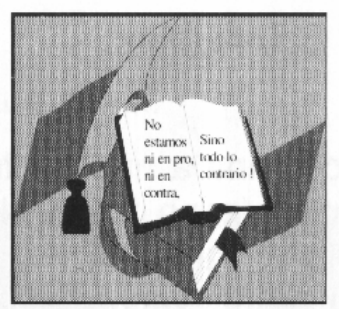

$\mathrm{L}$

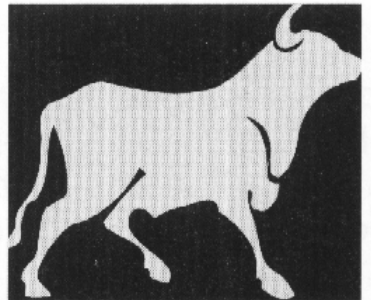

$\mathrm{T}$

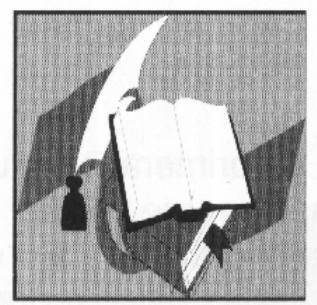

Q

Figura 6.

Con esta definición de contenencia, las $\underline{n}$-pinturas $\mathrm{V}$ y $\mathrm{F}$ de la figura 3 , que son $\underline{n}$-pinturas para cualquier valor de $n$, se comportan como los conjuntos universal (X) y vacío $(\varnothing)$ de la teoría de conjuntos en el sentido de que toda $\underline{n}$-pintura A está contenida en la $\underline{n}$-pintura $\mathrm{V}$ y $\mathrm{F}$ está contenida en toda $\underline{n}$-pintura. Esto es, para toda $\underline{n}$-pintura $\mathrm{A}$ y todo valor de $n$, se tiene que $\mathrm{F} \leq \mathrm{A} \leq \mathrm{V}$.

\section{Operaciones entre $\underline{n}$-pinturas}

Análogamente a lo que sucede en teoría de conjuntos cuando se definen las operaciones de unión, intersección, diferencia, diferencia simétrica y complementación entre ellos, utilizando los conectivos lógicos de disyunción, conjunción, implicación, equivalencia y negación, podemos utilizar el orden definido entre $\underline{n}$-pinturas para generar operaciones entre ellas que sean generalizaciones de las generadas en los conjuntos por los conectivos de la lógica bivalente.

Con este propósito, observamos que los conectivos lógicos pueden obtenerse del orden del conjunto $\underline{2}=\{0,1\}$ así:

Dado un conjunto $X$, considerado como conjunto referencial, $A$ y $B$ subconjuntos de él, para cada $x \in X$ se tiene que:

i. El valor de verdad de $(x \in(A \cup B))$, es el mayor de los valores de verdad de las proposiciones $(x \in A)$ y $(x \in B)$.

ii. El valor de verdad de $(x \in(A \cap B))$, es el menor de los valores de verdad de las proposiciones $(x \in A)$ y $(x \in B)$.

iii. El valor de verdad de $(x \in(A \rightarrow B))$, es 1 si el valor de verdad de la proposición $(x \in A$ ) es menor o igual que el valor de verdad de la proposición $(x \in B$ ) y es el valor de verdad de la proposición $(x \in B)$ en caso contrario. 
iv. El valor de verdad de $(x \in(A \leftrightarrow B))$, es el menor de los valores de verdad entre $(x \in(A \rightarrow B)$ y $(x \in(B \rightarrow A))$. $0))^{1}$.

v. El valor de verdad de $\left(x \in A^{c}\right)$, es el valor de verdad de la proposición $(x \in(A \rightarrow$

Por tanto una generalización de estas operaciones a n-pinturas puede obtenerse [8] del orden existente entre las tonalidades de colores seleccionadas así:

Dadas dos $\underline{n}$-pinturas A y B, definimos:

1. La intersección de $A$ y $B$ que notamos $A \wedge B$ como la $\underline{n}$-pintura que se obtiene de asignar a cada punto de la hoja la tonalidad más oscura de las presentes en cada una de ellas o igual .si las dos coinciden

2. La unión de $\mathrm{A}$ y $\mathrm{B}$ que notamos $\mathrm{A} \vee \mathrm{B}$ como la $\underline{n}$-pintura que se obtiene de asignar a cada punto de la hoja la tonalidad más clara de las presentes en cada una de ellas, 0 igual si las dos coinciden.

3. La codiferencia de $\mathrm{A}$ y $\mathrm{B}$ que notamos $\mathrm{A} \rightarrow \mathrm{B}$ como la $\underline{n}$-pintura que se obtiene de asignar a cada punto de la hoja la tonalidad más clara de todas las posibles, si en $A$ es más oscura o igual que en $\mathrm{B}$; el color en $\mathrm{B}$ si no es así.

4. La codiferencia simétrica de $A$ y $B$ que notamos $A \leftrightarrow B$ como la $\underline{n}$-pintura resultante de intersectar $A \rightarrow B \quad$ y $B \rightarrow A$. $A \rightarrow F$.

5. El negativo de A que notamos $\neg$ A como la $\underline{n}$-pintura resultante de la operación

Veamos algunas ilustraciones de estas operaciones en el caso de tres tonalidades de grises:

Si A y B son las $\underline{3}$-pinturas que se muestran en la figura 7 .

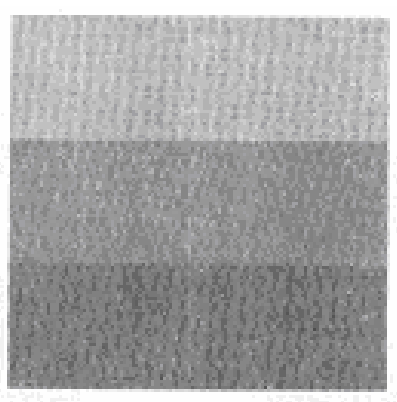

A

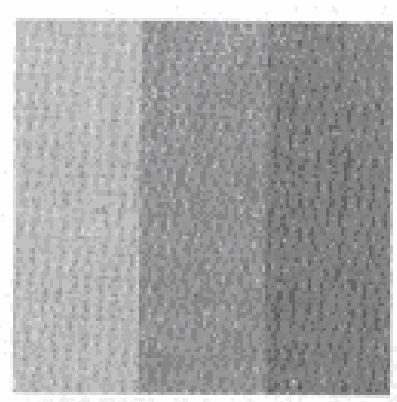

B

Figura 7.

\footnotetext{
1 Esta es una forma alternativa de presentar la negación de una proposición cualquiera, debido a que $\sim(p$
} $\rightarrow q)=p \wedge \sim q$, de donde $p \rightarrow q=p \vee q$ ysi $q$ es falso $(F)$ entonces $p \rightarrow F=\sim p$. 
Su intersección y su unión son respectivamente las 3-figuras:

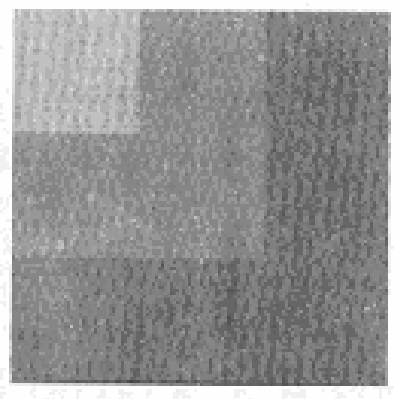

$A \wedge B$

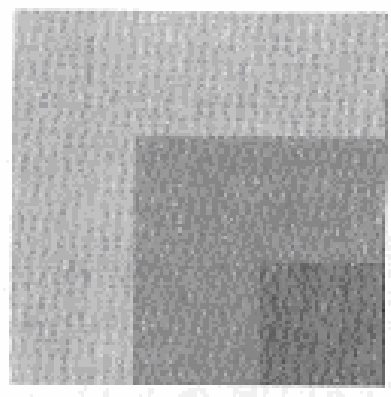

AvB

Figura 8.

Su codiferencia y su codiferencia simétrica se muestran en la figura 9.

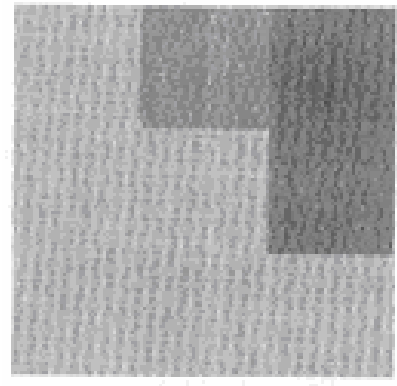

$\mathrm{A} \rightarrow \mathrm{B}$

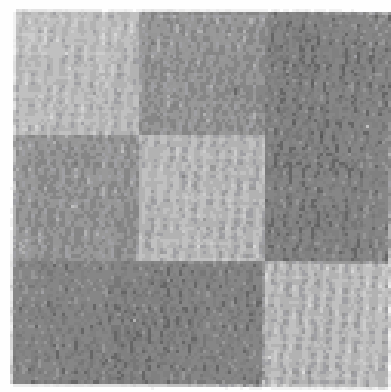

$A \leftrightarrow B$

Figura 9.

Los negativos de las $\underline{3}$-pinturas A y B se presentan en la figura 10 , los cuales por su definición, sin importar que tan amplia sea la gama de tonalidades que se escoja, siempre serán $\underline{n}$-pinturas en sólo dos tonalidades, la más clara y la más oscura de las tonalidades posibles.

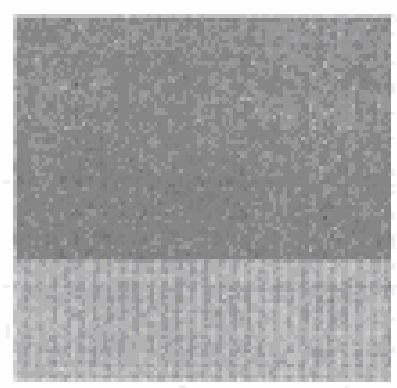

$$
\mathrm{A} \rightarrow \mathrm{F}=\neg \mathrm{A}
$$

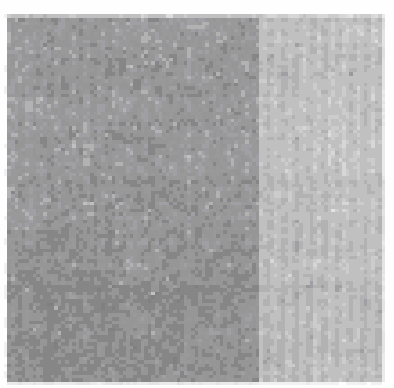

$$
\mathrm{B} \rightarrow \mathrm{F}=\neg \mathrm{B}
$$

Figura 10.

Cuando se reduce el estudio a dos tonalidades se tienen las operaciones de la teoría usual de conjuntos y los diagramas resultan similares a los de Euler-Venn, resaltando que: 
i. El color más claro representa a los elementos que forman parte del conjunto.

ii. La codiferencia y la codiferencia simétrica corresponden a los complementos de la diferencia entre $A$ y $B(A-B)$ y de la diferencia simétrica $(A \Delta B)$, lo que motivó la elección de los nombres.

iii. El negativo corresponde al complemento, aunque en el caso general éste es tan sólo un pseudocomplemento [5] debido a que satisface que $A \wedge \neg A=F$ pero no que $A \wedge \neg$ $\mathrm{A}=\mathrm{V}$ como se presenta en la figura 11 para el caso de la $\underline{3}$-pintura $\mathrm{B}$.

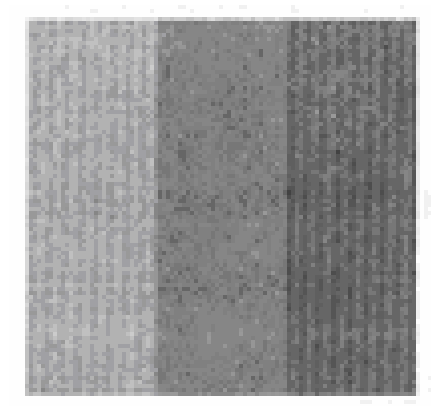

B

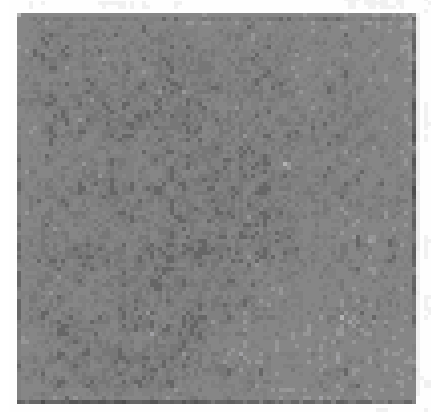

$\mathrm{B} \wedge \neg \mathrm{B}$

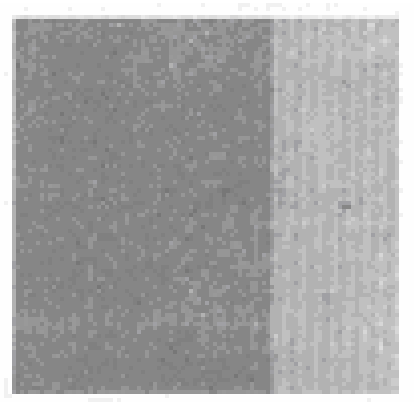

$\rightarrow B$

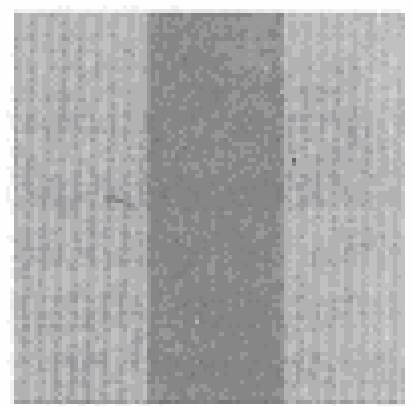

$B \vee \neg B$

Figura 11.

\section{Operaciones entre $\underline{n}$-predicados}

Si identificamos con $\mathrm{p}(\mathrm{x})$ y $q(\mathrm{x})$ a los $\underline{n}$-predicados que definen las $\underline{n}$-pinturas $\mathrm{A}$ y $\mathrm{B}$ respectivamente y recordamos que, teniendo un universo como referencia, cada $\underline{n}$-pintura representa un $\underline{n}$-predicado y viceversa, podemos hacer corresponder a cada operación entre las $\underline{n}$-pinturas una operación entre $\underline{n}$-predicados de acuerdo con la siguiente tabla:

\begin{tabular}{|l|l|}
\hline \multicolumn{2}{|c|}{ OPERACIONES } \\
\hline$\underline{n}$-pinturas & $\underline{n}$-predicados \\
\hline Intersección & Conjunción \\
Unión & Disyunción \\
Codiferencia & Implicación \\
Codiferencia Simétrica & Equivalencia \\
Negativo & Seudonegación \\
\hline
\end{tabular}


Por ejemplo la conjunción de los predicados $p(x)$ y $q(x)$, notado $(p \wedge q)(x)$, está definido para cada $x \in X$ por la tonalidad correspondiente al punto $x$ en la $\underline{n}$-pintura $A \wedge B$.

\section{7. $\underline{n}$ - proposiciones}

Llamaremos $\underline{n}$-proposición a la frase que nos permite seleccionar una tonalidad en una n-pintura; del resultado de esta elección diremos que es un valor de verdad, debido a que este proceso corresponde en lógica bivalente a la elección de un valor de verdad (verdadero $(\mathrm{V})$ o Falso $(\mathrm{F})$ ) de los posibles para un predicado en un universo de referencia preestablecido.

Esta selección puede hacerse por diversos caminos, algunas alternativas son las siguientes:

1. Escogiendo el color de un punto $x_{0}$ de una $\underline{n}$-pintura dada. Esto es el equivalente en la lógica clásica a la obtención de proposiciones $\mathrm{p}\left(\mathrm{x}_{0}\right)$ a partir de un predicado $\mathrm{p}(\mathrm{x})$, evaluándolo en un elemento $\mathrm{x}_{0}$ del conjunto universal.

2. Escogiendo la tonalidad más clara utilizada en toda la $\underline{n}$-pintura. Esta selección de tonalidad garantiza la presencia de por lo menos un punto con esa tonalidad, junto con la ausencia de una tonalidad más clara en toda la pintura. Tomaremos esta escogencia como definición del cuantificador existencial (Э), generalizado a $\underline{n}$-pinturas, puesto que cuando nos restringimos a solo dos tonalidades, esta elección resulta de la tonalidad más clara (que identificamos con el valor verdadero) si existe algún punto de la hoja con esa tonalidad y es de la tonalidad más oscura (que identificamos con el valor falso) si toda la hoja tiene esa tonalidad.

3. Escogiendo la tonalidad más oscura utilizada en toda la $\underline{n}$-pintura, obtenemos la definición para el cuantificador universal (V) generalizado a $\underline{n}$-pinturas, garantizando que hay un punto con esa tonalidad y la ausencia de una tonalidad más oscura, puesto que cuando nos restringimos a dos tonalidades, la elección tiene valor verdadero cuando toda la hoja está pintada del color más claro y tiene valor falso si hay por lo menos un punto con la tonalidad más oscura.

Estos no son las únicas opciones para seleccionar una tonalidad de una $\underline{n}$-pintura. Otras posibilidades se presentan aplicando los cuantificadores definidos a cualquier zona (subconjunto de $\mathrm{X}$ ) de la $\underline{n}$-pintura. Es así como podría escogerse la tonalidad más clara utilizada en Tos ojos de la Monalisa, suponiendo que esta zona está bien delimitada.

En la vida cotidiana se presentan situaciones que usualmente son descritas con afirmaciones que solo tienen dos posibilidades extremas: Sucio o limpio, bueno o malo, bello o feo, etc. Sin embargo, ampliando la gama de posibilidades utilizando algún criterio preestablecido, estas podrían describirse mejor.

Por ejemplo, si el universo es un conjunto de seres humanos, el predicado " $x$ es sagaz" genera una división del universo en tantas partes como opciones de clasificación de lo sagaz tengamos. Y si logramos una lista de comportamientos que permitan clasificarlo en $n$ estados, podríamos hacer el mapa de sagacidad de la población, el cual correspondería a una $\underline{n}$-pintura, de tal manera que si logramos establecer principios de razonamiento válidos utilizando $\underline{n}$-pinturas, tendremos principios de razonamiento válidos para lógicas con dos o más valores, tarea que abordaremos enseguida. 


\section{Tautologías}

Dado un conjunto de $\underline{n}$-pinturas, es posible definir operaciones entre ellas cuyo resultado sea una $\underline{n}$-pintura de una sola tonalidad, que en particular puede ser la $\underline{n}$-pintura $\mathrm{V}$ o la F. Por ejemplo si R y S son las $\underline{3}$-pinturas:

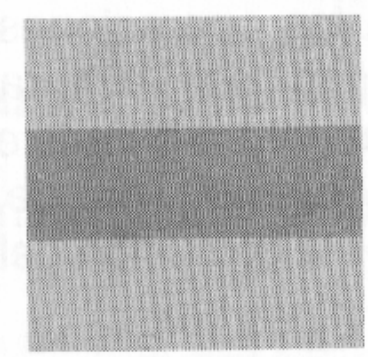

R

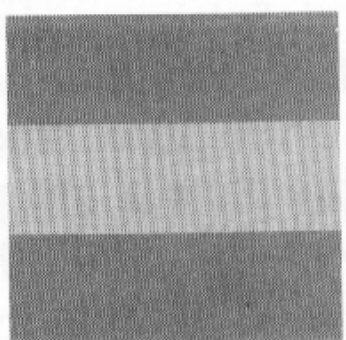

S

Figura 12.

Su unión es la $\underline{3}$-pintura $V$, pero esto naturalmente no es válido para cualquier par de n-pinturas.

Sin embargo, existen operaciones entre n-pinturas cuyo resultado es una hoja de una sola tonalidad, sin importar las $\underline{n}$-pinturas que se utilicen para efectuar la operación, por ejemplo:

La operación

$$
(A \wedge B) \rightarrow A
$$

tiene como resultado la $\underline{n}$-pintura $\mathrm{V}$, independiente de las $\underline{n}$-pinturas A y $\mathrm{B}$ que se escojan para ser operadas, como puede verificarse en el caso particular siguiente:

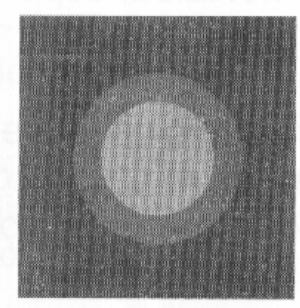

A

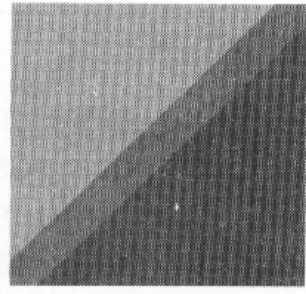

B

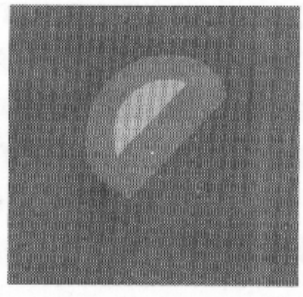

$(A \wedge B)$

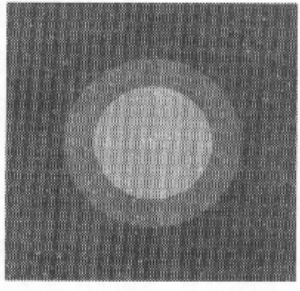

A

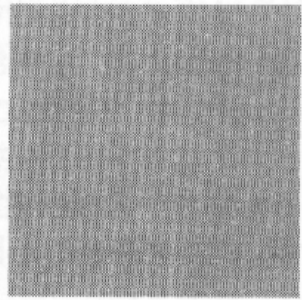

$(A \wedge B) \rightarrow A=V$

Figura 13 
Esta verificación en uno o varios casos particulares no constituye una demostración, pero nos permite acercarnos de manera intuitiva a la validez de la afirmación realizada, lo que constituye uno de nuestros propósitos fundamentales.

Una demostración deberá hacerse en términos de $\underline{n}$-pinturas y tonalidades arbitrarias, utilizando las definiciones dadas para las operaciones. Por ejemplo, en el caso que nos ocupa debemos observar que dadas dos $\underline{n}$-pinturas $\mathrm{A}$ y $\mathrm{B}$ cualesquiera, la tonalidad que tiene en un punto elegido de manera arbitraria en la $n$-pintura $(A \wedge B)$ es más clara que la que tiene el mismo punto en cualquiera de las $\underline{n}$-pintu ras $A$ y $B$, (por la definición de $(A \wedge B)$ ), esto significa, por la definición del orden entre $\underline{n}$-pinturas, que

$$
(A \wedge B) \leq A
$$

y por lo tanto la tonalidad del punto en la $\underline{n}$-pintura

$$
(A \wedge B) \rightarrow A
$$

es el más claro de todos los posibles (por ja definición de $X \rightarrow Y$ ), lo que da como resultado la $\mathrm{n}$-pintura que hemos notado $\mathrm{V}$.

En el lenguaje de $\underline{n}$-predicados, diremos que si $\mathrm{p}(\mathrm{x})$ y $q(x)$ son dos $\underline{n}$-predicados cualesquiera, asociados con a-pinturas $A$ y $B$ respectivamente el $\underline{n}$-predicado

$$
((p(x) q(x)) \rightarrow p(x))
$$

que es el asociado a la $\underline{n}$-pintura

$$
(A \wedge B) \rightarrow A
$$

asigna la tonalidad más clara en todos los puntos de la hoja y por lo tanto da lugar a una proposición que escribiremos simbólicamente:

$$
(\forall x)((p(x) \wedge q(x)) \rightarrow p(x))
$$

la cual tiene el valor de verdad de la tonalidad más clara (verdadero) independiente de las n-pinturas que se escojan para hacer la operación.

Las proposiciones que tengan esta característica las llamaremos tautologías.

Sí de manera similar se puede construir proposiciones a partir de operaciones entre n-pinturas en las que sin importar cual sea el conjunto inicial de a-pinturas el resultado sea una hoja de una sola tonalidad, no necesariamente la más clara (digamos la tonalidad asociada con $k$ ), las llamaremos k-tautologías. En particular a las O-tautologías las llamaremos contradicciones y las (n-1)-tautologías simplemente tautologías.

\section{Razonamientos}

Toda tautología de la forma 


$$
\forall x(p(x) \rightarrow q(x))
$$

la llamaremos un razonamiento válido.

Así mismo, llamaremos un $\boldsymbol{k}$-razonamiento a la proposición

$$
\forall x(p(x) \rightarrow q(x))
$$

si para cualquier par de u-predicados $p(x)$ y $q(x)$ es ella una $k$-Tautología.

Usaremos de nuevo las u-pinturas A y B de la figura 13, que suponemos asociadas con los predicados $p(x)$ y $q(x)$ respectivamente, para intuir razonamientos válidos y para encontrar razonamientos que siendo válidos en la lógica clásica, no lo son cuando consideramos más valores de verdad. La demostración de la validez de un razonamiento requiere, como antes, la consideración de todas las tonalidades y todas las $\underline{n}$-pinturas, pero la demostración de su no validez sólo requiere de un contraejemplo. En particular usaremos $\underline{3}$-pinturas para ilustrar nuestras afirmaciones.

1. Hemos observado que la 3-pintura $(A \wedge B) \rightarrow A$ tiene la tonalidad más clara en todos sus puntos y se ha demostrado que esto es válido cualesquiera que sean las $\mathrm{n}$-pinturas $\mathrm{A}$ y $\mathrm{B}$ seleccionadas, por lo tanto el razonamiento

$$
\forall x((p(x) \wedge q(x)) \rightarrow p(x))
$$

conocido como la ley de eliminación es válido.

2. Otra forma clásica de razonamiento que se encuentra en la base de todas las demostraciones en matemáticas es conocida como el modus ponendo ponens, ella se utiliza para inferir la verdad de una proposición a partir de una implicación.

En la figura 14, se representa la secuencia de operaciones que conducen a la $n$-pintura $((A \rightarrow B) \wedge A) \rightarrow B$ asociada con la proposición

$$
\forall x(((p(x) \rightarrow q(x)) \wedge(p(x)) \rightarrow q(x))
$$

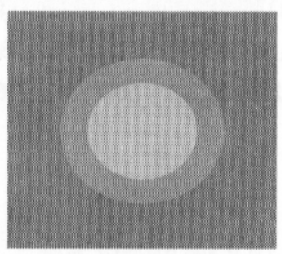

A

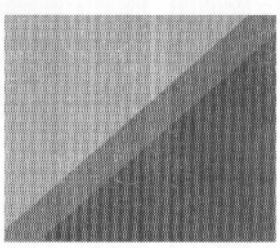

A

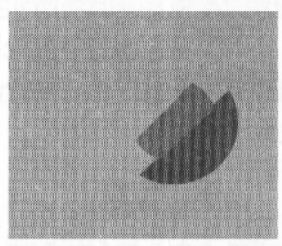

$A \rightarrow B$

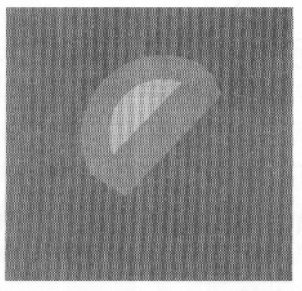

$A \wedge(A \rightarrow B)$

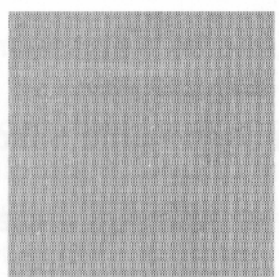

$((\mathrm{A} \rightarrow \mathrm{B}) \wedge \mathrm{A}) \rightarrow \mathrm{B}$

Figura 14. 
sugiriéndonos que el razonamiento es válido también cuando se consideran tres valores de verdad en correspondencia con las tres tonalidades de grises presentadas.

Una demostración de su validez para el caso de $n$ tonalidades, tiene la siguiente forma:

Sea $x$ un punto arbitrario de un universo X, A y B dos n-pinturas en X. Analicemos dos casos:

Si la tonalidad que tiene el punto $x$ en la $\underline{n}$-pintura $A$ es más oscuro o igual a la tonalidad que tiene en la $\underline{n}$-pintura $\mathrm{B}$, el color del punto en $\mathrm{A} \rightarrow \mathrm{B}$ es el correspondiente a la tonalidad más clara de todas las posibles y por lo tanto al compararla con la tonalidad del punto en $A$ con la operación intersección, en $(A \rightarrow B) \wedge A$ nos da como resultado el color que tiene en $A$. Por consiguiente, la tonalidad que tiene el punto $x$ en la $\underline{n}$-pintura ((A $\rightarrow B) \wedge A$ ) $\rightarrow$ B será la tonalidad más clara de todas las posibles.

Si por el contrario, la tonalidad que tiene el punto $x$ en la n-pintura $A$ es más Clara que la que tiene en la $\underline{n}$-pintura $\mathrm{B}$, el color del punto en $\mathrm{A} \rightarrow \mathrm{B}$ es el correspondiente a la tonalidad que tenga en la n-pintura $B$ y por lo tanto al compararla con la tonalidad del punto en $A$ con la operación intersección, en $((A \rightarrow B) \wedge A)$ nos da como resultado el color que tiene en $B$. Por consiguiente, la tonalidad que tiene el punto $x$ en la $\underline{n}$-pintura $((A \rightarrow B)$ A) $\rightarrow$ B será la tonalidad más clara de todas las posibles.

3. Un razonamiento que es válido en la lógica clásica es el principio del tercio excluso, el cual afirma que una proposición cualquiera o su negación, siempre es verdadera. Este sin embargo no es válido cuando se consideran tres o más valores le verdad, asociados con diferentes tonalidades de un mismo color, como lo garantiza el ejemplo representado en la figura 15, correspondiente a la $\underline{3}$-pintura $A \vee \neg A$, la cual no coincide con la $\underline{3}$-pintura V.

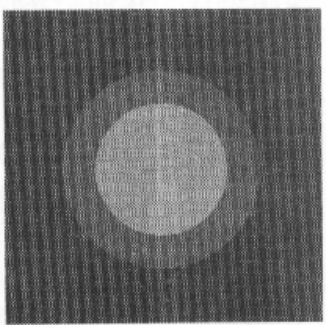

A

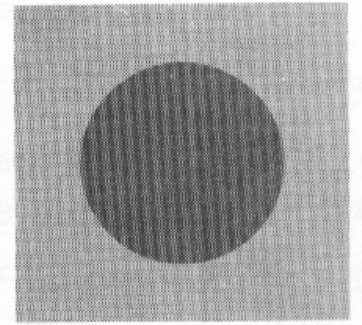

$\neg \mathrm{A}$

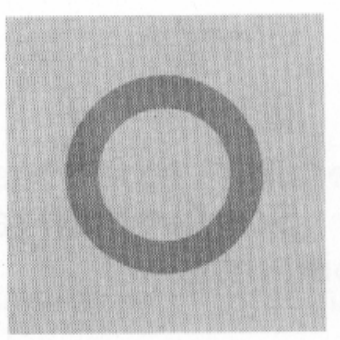

$A \vee \neg A \neq V$

Figura 15

Por tanto la proposición $\forall x(p(x) \vee \neg p(x))$ no es un razonamiento válido.

4. A pesar de que la seudonegación no tiene el mismo comportamiento que la legación de la lógica clásica, como puede verse en la ley del tercio excluso, las proposiciones. 


$$
\begin{aligned}
& \forall x(\neg(p(x) \wedge q(x)) \rightarrow(\neg p(x) \vee \neg q(x)) \\
& \forall x((\neg(p(x) \vee \neg q(x)) \rightarrow(\neg(p(x) \vee q(x)) \\
& \forall x(\neg(p(x) \vee q(x)) \rightarrow(\neg p(x) \vee \neg q(x)) \\
& \forall x \quad(\neg(p(x) \wedge \neg q(x)) \rightarrow(\neg p(x) \vee q(x))
\end{aligned}
$$

que son equivalentes a las leyes de De Morgan, son también razonamientos válidos cuando se consideran valores de verdad asociados con varias tonalidades de un mismo color, como lo sugieren los diagramas de la figura 16.

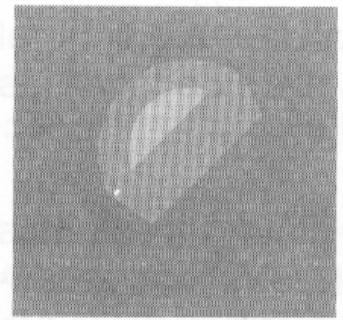

$A \wedge B$

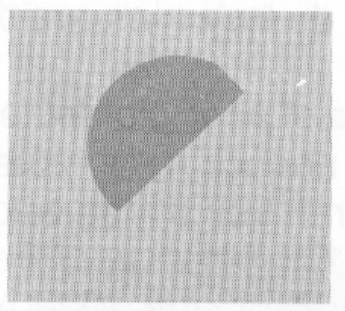

$\neg(A \wedge B)$

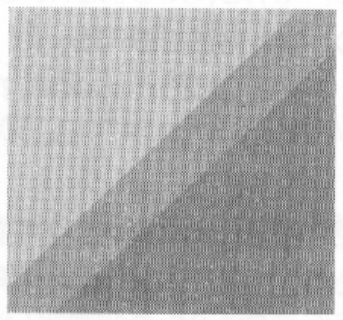

B

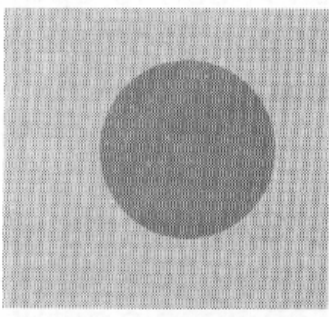

$\neg \mathrm{A}$

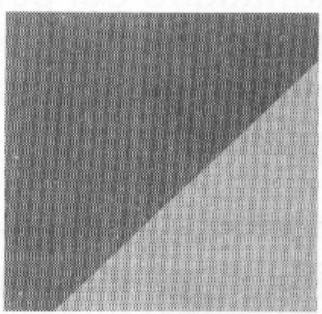

$\neg \mathrm{B}$

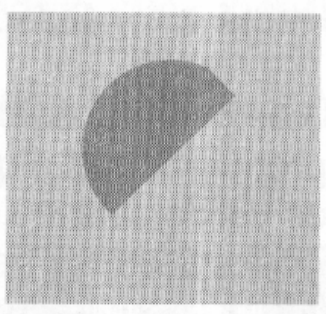

$\neg \mathrm{A} \vee \neg \mathrm{B}$

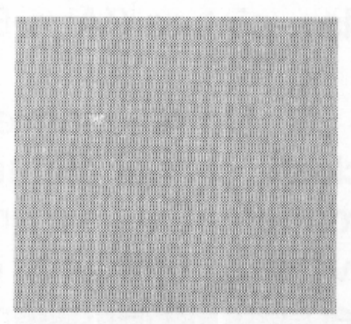

$\neg(\mathrm{A} \wedge \mathrm{B}) \rightarrow(\neg \mathrm{A} \vee \neg \mathrm{B})$

Figura 16.

Son también razonamientos válidos, que sugerimos intuya a partir de las $\underline{n}$-pinturas:

Ley de Agregación: $\forall x(p(x) \rightarrow(p(. x-) \vee q(x)))$.

Principio de no-contradicción: $\forall x(\neg(p(x) \wedge \neg p(x)))$.

Ley de casos: $\forall x(p(x) \rightarrow q(x)) \wedge(p(x) \rightarrow q(x))) \rightarrow((p(x) y p(x)) \rightarrow q(x)))$.

Ley del absurdo: $\forall x(p(x) \rightarrow q(x)) \wedge(p(x) \rightarrow \neg q(x)) \rightarrow \neg p(x))$

Se tiene solamente una de las implicaciones de la ley de doble negación:

$$
\forall x(p(x) \rightarrow(\neg \neg, p(x)))
$$

Son ejemplos de razonamientos válidos en la lógica clásica, que se puede comprobar mediante diagramas, que no son válidos en lógicas con más de dos valores de verdad como las construidas a partir de las tonalidades:

La otra implicación de la ley de doble negación: $\forall x(\neg(\neg p(x)) \rightarrow, p(x))$. 
Ley de la contrapositiva: $\forall x(\neg \mathrm{q}(\mathrm{x}) \rightarrow(\neg \mathrm{p}(\mathrm{x})) \rightarrow(\mathrm{p}(\mathrm{x}) \rightarrow \mathrm{q}(\mathrm{x})))$

Ley de reducción al absurdo: $\left.\forall x(p(x) \wedge \neg q(x)) \rightarrow\left(p(x)^{\wedge} \neg p(x)\right) \rightarrow(p(x) \rightarrow q(x))\right)$.

Hasta aquí hemos presentado una manera para aproximarnos intuitivamente a razonamientos válidos en lógicas no clásicas, utilizando inicialmente las n-pinturas y el orden existente entre las tonalidades de un mismo color para definir operaciones entre ellas con el fin de asociar con cada n-pintura un n-predicado y con cada operación un conectivo lógico que nos permite construir proposiciones con tantos valores de verdad como tonalidades. Con estas herramientas podemos identificar razonamientos válidos dentro de una lógica así construida, reconociendo resultados de operaciones que dejan la hoja de la tonalidad más clara, sin importar las n-pinturas de partida. Consideramos que este tratamiento es susceptible de ser llevado al aula con la intención de facilitar el acercamiento de los estudiantes a la lógica intuicionista.

\section{BIBLIOGRAFIA}

[1] BARNES, D. y MACK, J. An algebraic introduction to Mathematical Logic. SpringerVerlag. New York. 1975.

[2] CAICEDO, $X$. Elementos de Lógica y calculabilidad. Una empresa Docente. Universidad de los Andes. Santafé de Bogotá, 1990.

[3] COPI, I. M. Lógica Simbólica. Ed. Continental. México. 1979.

[4] GOLDBLATT, R. Topoi: A Categorical analysis of Logic. Elsevier Science Publishers. New York. 1984.

[5] GRATZER, S. Lattice theory: First concepts and distributive lattices. W.H. Freeman. U.S.A. 1971.

[6] KAUFMANN, A. Introducción a la teoría de subconjuntos borrosos. C.E.C.S.A. México. 1977.

[7] LUQUE, C.; DONADO, A. y PÁEZ, J. Caracterización de conjuntos por temas. XIII Coloquio Distrital de Matemáticas y estadística. Universidad Nacional. Santafé de Bogotá, 1996.

[8] LUQUE, C.; PÁEZ, J.; DONADO, A. H-conjuntos: Una generalización de la noción de conjunto. XIV Coloquio Distrital de Matemáticas y estadística. Universidad Pedagógica Nacional. Santafé de Bogotá, 1997.

[9] STOLL, Robert. Set theory and logic. W. H. Freeman. U.S.A. 1963. 